\title{
HST OBSERVATIONS OF THE GLOBULAR CLUSTER M4
}

\author{
G. G. FAHLMAN
}

Department of Geophysics and Astronomy, University of British Columbia, Vancouver, B.C., Canada V6T 1Z4

H.B. RICHER, R.I. IBATA, N.C. IVANANS, G. MANDUSHEV

Department of Geophysics and Astronomy, University of British Columbia.

J.E. HESSER, P.B. STETSON

Dominion Astrophysical Observatory, Herzberg Institute of Astrophysics, National Research Council. M. A. WOOD

Department of Physics and Space Science, Florida Institute of Technology

\section{D.A. VANDENBERG}

Department of Physics and Astronomy, University of Victoria.

C. PRYOR

Department of Physics and Astronomy, Rutgers University. W.E. HARRIS

Department of Physics and Astronomy, McMaster University.

H.E. BOND

Space telescope Science Institute.

M. BOLTE

Lick Observatory, University of California.

AND

R.A. BELL

Department of Astronomy, University of Maryland.

\footnotetext{
Abstract.

The WFPC2 aboard the Hubble Space Telescope has been used to obtain deep images in three fields at different radial positions in the nearest 
Globular cluster, M4 (NGC 6121). In this paper, we discuss the white dwarf cooling sequence and show how the dynamical structure of the cluster will affect their cumulative distribution function. We also present the first discussion of our observations of the faint cluster main sequence stars.

\section{Introduction}

The high angular resolution achievable with the Hubble Space Telescope offers two significant advantages over ground based observations of globular star clusters: (1) accurate photometry to faint levels is feasible in crowded cluster cores, and (2) the improved contrast of the sharper stellar profile against a lower and much more stable background allows photometry of very faint stars. By exploiting both of these improvements, we are able to study two stellar populations, the cluster white dwarfs and the low mass main sequence stars, which have proven inaccessible from the ground. These stars play key roles in the dynamical evolution of the clusters and provide important data pertaining to stellar evolution and star formation in the early universe.

Messier 4 (NGC 6121), at a distance of only $2.0 \mathrm{kpc}$, is the globular cluster closest to the sun. In this paper, we will describe our multi-field observational program for M4. At the time of this meeting, we have completed and calibrated most of the photometry for our program fields but a full analysis of these results, which require extensive computations to determine the completeness corrections and photometric error distributions has not yet been done. Previous discussion of the white dwarfs can be found in Richer et al. (1995a; paper I) and Richer et al. (1995b; paper II) discuss the implications of our photometry on the issue of the binary fraction in M4.

\section{Observations and Data Reduction}

The data described here was acquired under the HST proposal number 5461 and the STScI electronic information service can be used to obtain full details of the program.

Images were obtained with the WFPC2 camera through $U(\mathrm{~F} 336 \mathrm{~W})$, $V(\mathrm{~F} 555 \mathrm{~W})$ and $I(\mathrm{~F} 814 \mathrm{~W})$ filters in two inner fields whose position in the cluster was specified by the projected location of the PC chip. One of these fields was located within the cluster core (referred to as the core field) and the other was located at 1 core radius. (These positions are based on an adopted core radius of $r_{c}=74^{\prime \prime}$ from Webbink 1985.) The principal goal for these observations was to study the white dwarf cooling sequence in M4. 
The outer field was located at 4 core radii and only $V$ and $I$ images were obtained. The goal in this relatively uncrowded field was to get sufficiently deep to see the end of the cluster main-sequence.

The raw data frames had the standard HST pipeline processing applied to them as discussed in paper I. The photometry was carried out with the ALLFRAME package developed by Stetson (1994). The calibration of the data follows the methodology described by Holtzman et al. (1995) and is therefore placed on the ground-based $U, V, I$ system defined by the Landolt (1992) standards. We assume the reddening law specified by the following coefficients: $\mathrm{R}=\mathrm{A}_{\mathrm{V}} / \mathrm{E}(\mathrm{B}-\mathrm{V})=3.12, \mathrm{E}(\mathrm{V}-\mathrm{I})=1.25 \mathrm{E}(\mathrm{B}-\mathrm{V}), \mathrm{E}(\mathrm{U}-$ $\mathrm{I})=2.98 \mathrm{E}(\mathrm{B}-\mathrm{V})$ and $\mathrm{A}_{\mathrm{U}}=4.83 \mathrm{E}(\mathrm{B}-\mathrm{V})$ (Bessell and Brett 1988, Mathis 1990). The apparent distance modulus and cluster reddening adopted are $(\mathrm{m}-\mathrm{M})_{\mathrm{V}}=12.65$, and $\mathrm{E}(\mathrm{B}-\mathrm{V})=0.37$, respectively (see paper I for justification of these choices).

We have completed the photometric reductions for all the data in the 1 core and 4 core fields. For the core field, we have reduced data for only a single WFC chip and the PC chip. The remaining data has a significant overlap with the 1 core field data and all will be simultaneously reduced with ALLFRAME. We will limit the disçussion in this paper to the 1 core and 4 core data only.

\section{White Dwarfs}

The white dwarf cooling sequence is most clearly delineated on the $(U, U$ I) plane, shown in figure 1. The PSF fitting radius was set to a relatively large value so that the brighter, saturated stars were measured by fitting to the profile wings. The errors for these stars are large, accounting for the evident spread seen in figure 1. The turn-off in this color-magnitude plane has been observed from the ground and this shows that the photometry of the saturated stars has a bias as well as large errors in that the stellar locus for $\mathrm{M}_{\mathrm{U}} \leq 7.0$ appears to be too red. The faintest stars have an apparent magnitude of $U \geq 26$, more than a magnitude below the very deep ground based data obtained by Richer and Fahlman (1988) at the CFHT for their study of the cluster M71. In paper I, we have shown that the white dwarf sequence is consistent with the cooling locus of $0.5 \mathrm{M}_{\odot}$ carbon white dwarfs and this is illustrated in figure 1. where this model cooling curve has been overlayed on the data without adjustment.

If it is assumed that stars are conserved through their post-main-sequence (pms) evolutionary phases, then the number of wds brighter than a given magnitude, $\mathrm{N}(<M)$, should be proportional to the time taken to cool to that magnitude. In figure 2 , we show the cumulative luminosity function (CLF), $\mathrm{N}\left(<M_{U}\right)$, for the 1 core field. This is the only data for which 


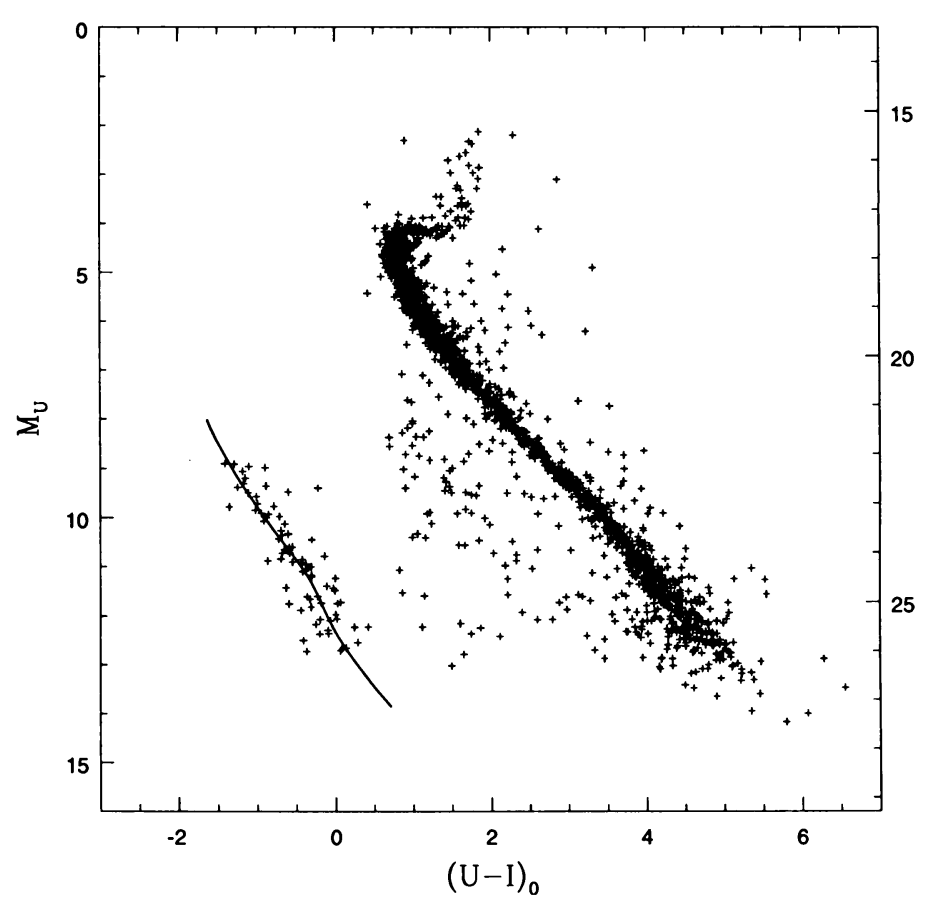

Figure 1. This CMD contains data from all four chips in the 1 core field of M4. The model curve is the cooling locus for pure carbon core white dwarfs with a mass $M=0.5 \mathrm{M}_{\odot}$. The right hand scale shows the apparent $U$ magnitude.

we have reasonable completeness corrections at present and the corrected counts, obtained by assigning a weight, equal to the completeness factor appropriate to the magnitude and CCD chip, to each observed star are also plotted. The three panels show theoretical curves for different interior compositions and are based on interior models developed by Matt Wood, and atmospheres from Bergeron et al. (1996). The theoretical counts are normalized to the indicated number of horizontal branch (HB) stars which were assumed to have a lifetime of $10^{8} \mathrm{yr}$. For reference, the number of HB stars expected in the HST field is 14.5 based on actual counts from ground based images of the M4 core.

The agreement between theory and observation is good at the bright end of the cooling sequence but, at the faint end there is a discrepancy which persists irrespective of the wd core composition. The deviation sets in at magnitudes for which the relevant stars are easily visible on the images and thus it would be surprising if this disagreement were due solely to poorly determined incompleteness corrections. 


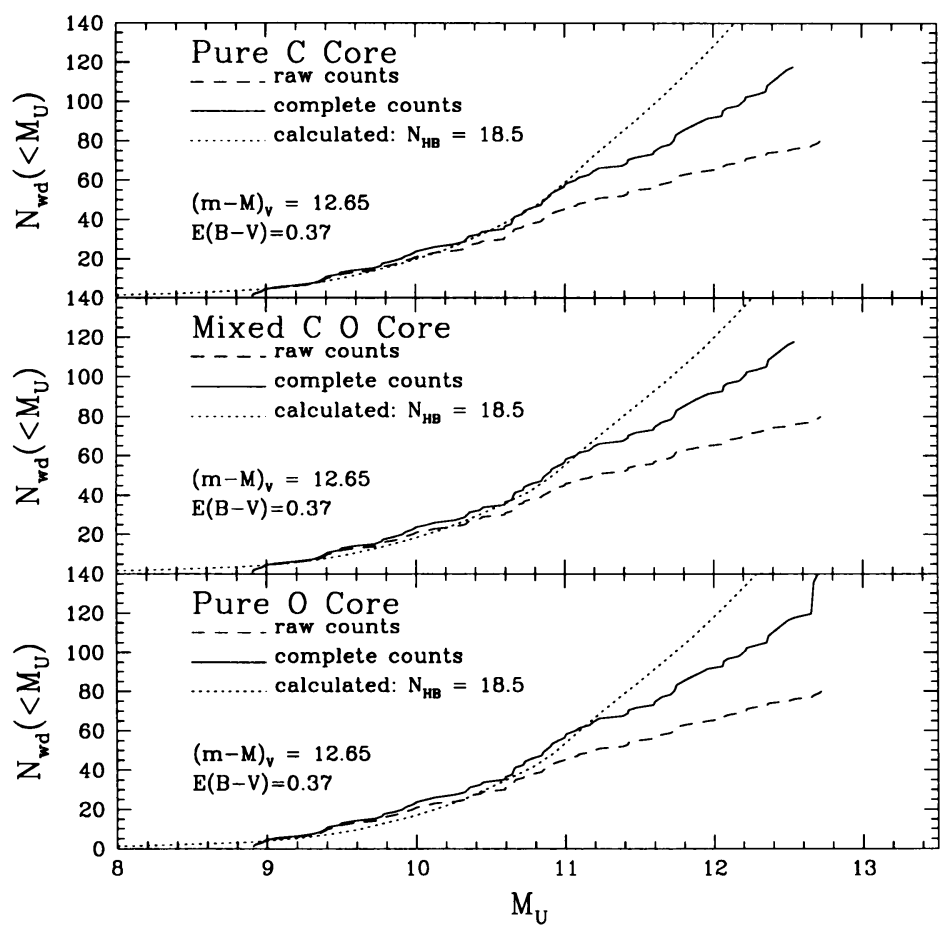

Figure 2. The observed cumulative luminosity function (CLF), both raw and completeness corrected, is compared to the theoretical CLF for white dwarf cooling sequences with three different interior compositions.

It appears that this disagreement could be due to mass segregation. The observed wds have a mass which is only $60 \%$ that of their progenitor stars and hence they will relax over time to a less concentrated spatial distribution than the cluster turn-off and pms stars we see today. The central and half-mass relaxation times in M4 are $4 \times 10^{7} \mathrm{yr}$ and $4 \times 10^{8} \mathrm{yr}$ respectively (Djorgovski 1993) whereas the white dwarfs with $M_{U} \geq 11.5$ have cooling times $\geq 5 \times 10^{8} \mathrm{yr}$. Hence the effects of relaxation should be apparent in the fainter and therefore older wds.

To illustrate the magnitude of the effect, we constructed an isotropic Michie-King model for M4. The present nuclear burning stars were assigned to 8 equal mass bins in the range $0.1-0.8 \mathrm{M}_{\odot}$ and the wd progenitors were assigned also to 8 equal mass bins in the range $0.8-8.0 \mathrm{M}_{\odot}$. An initial mass function (IMF) of the form $\mathrm{n}(\mathrm{m}) \propto \mathrm{m}^{-(1+\mathrm{x})}$ was adopted and, with $\mathrm{x}=1.25$, the mass fraction in each of the 16 mass bins was calculated. The upper 8 mass bins were then converted to wds according to the rule 


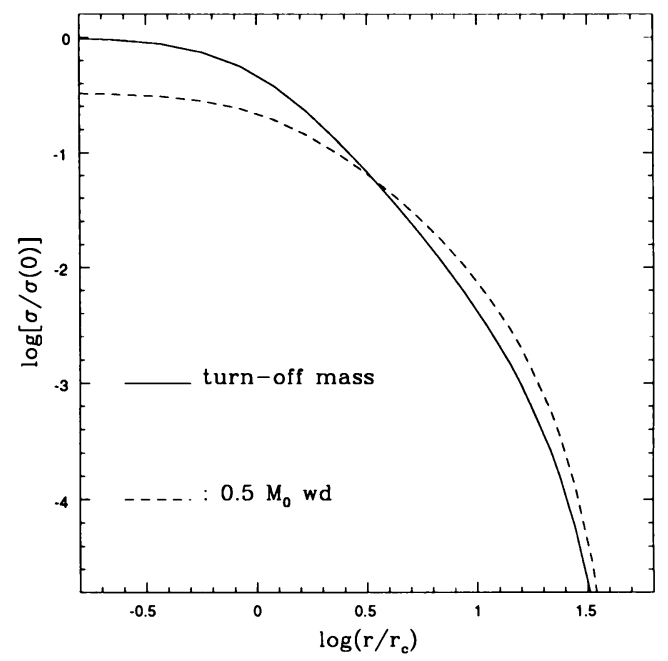

Figure 3. The normalized projected number density of turn-off stars is plotted as a iunction of cluster radius in a Michie-King model for M4. This profile is compared to the profile for $0.5 \mathrm{M}_{\odot}$ white dwarfs with the same mass fraction as the turn-off stars.

$\mathrm{m}_{w d}=\mathrm{m}_{o} \exp \left(\mathrm{m}_{i} / 8\right)$ as in Wood (1992). The constant $\mathrm{m}_{o}$ was set to give $\mathrm{m}_{w d}=0.5$ for $\mathrm{m}_{i}=1.17$, which is the number weighted mean mass (in $\mathrm{M}_{\odot}$ units) of the stars in the IMF bin just above the present turn-off. The central potential was then adjusted to give a model for which the stars in the turn-off mass bin gave a concentration parameter in good agreement with observation (Trager et al. 1995). In this model, stars identified with the turn-off constitute $2.6 \%$ of the cluster mass and the $0.5 \mathrm{M}_{\odot}$ wds contribute $15.2 \%$.

The normalized, projected number density of the model turn-off stars is plotted in figure 3 . This profile can be identified with the observed surface brightness profile and has a concentration parameter of $c=1.67$. We also show the profile of the $0.5 \mathrm{M}_{\odot}$ wds (dashed line) scaled to the same mass fraction as the turn-off stars. Essentially, the dashed line shows the effect of converting all the present turn-off stars to $0.5 \mathrm{M}_{\odot}$ wds and allowing them to fully relax to the quasi-equilibrium defined by the Michie-King model. As expected, the lower mass wds have a larger core radius than their progenitors. Close to the cluster core, the projected number density of wds is smaller than that of their progenitors and, therefore, smaller than the number which which would be predicted from the observed HB stars under the assumption of local star number conservation. The effect is reversed in the halo of the cluster. 
For the model shown here, the projected number of wds at 1 core radius is only $47 \%$ that of an equivalent progenitor population, which is similar to the observed discrepancy in the CLF of figure 2. Now this particular KingMichie model is meant to be illustrative only since the IMF is unconstrained and, more fundamentally, the model ignores the fact that the formation and relaxation processes are occurring on comparable time scales (more or less) for the stars which we can observe. In spite of these shortcomings, the model does demonstrate that mass segregation can have a significant effect on a locally measured wd CLF. The dynamical evolution can be treated in Fokker-Planck models which include stellar evolution, e.g., those of Drukier (1995) and these would be preferable to the static Michie-King models.

\section{The Main Sequence}

In the left panel of figure 4, we show a very high quality ground based CMD derived from data obtained in 1990 at the du Pont $2.5 \mathrm{~m}$ telescope of the Las Campanas Observatory (Thompson et al. 1990). This data was calibrated against Landolt standards and then transformed to the absolute CMD plane with the distance modulus and reddening quoted earlier. On the right, we have added the HST photometry for our 1 core and 4 core fields to give a CMD which spans the cluster m-s from the turn-off to within a few thousandths of a solar mass of the expected hydrogen burning cut-off. The excellent agreement between these data sets adds to our confidence in the Holtzman et al. calibration. The CMD for the HST data alone is shown in the left panel of figure 5 .

Our line of sight to $\mathrm{M} 4$, at $(1, \mathrm{~b})=(351,16)$, intersects the galactic bulge and, for a solar galacto-centric distance of $\mathrm{R}_{o}=8.0 \mathrm{kpc}$, passes the center of the Galaxy at a radius $\mathrm{R}=2.5 \mathrm{kpc}$. Hence, the stars in bulge should define a m-s ridge line some 2.9 mag below that of M4 if they have roughly the same metallicity. This is in good agreement with the stellar sequence visible immediately below and to the left of the cluster m-s. The bulge turn-off stars appear to have a color just slightly to the red of that of M4, indicating that they indeed have a similar metallicity and a similar age.

In the panel on the right of figure 5 , we have superimposed on the 4 core field CMD, a model isochrone from d'Antona and Mazzitelli (1995) that has a metallicity similar to that of M4. The stellar mass along the isochrone is also indicated with the last labeled point being the hydrogen burning limit. Clearly, this isochrone is a poor match to the lower m-s of M4. Although the gross morphology - the changes in slope along the m-s - do seem to be in rough agreement with the observations, the colors are too blue. The more limited models of Baraffe et al. (1995) show a similar discrepancy.

The physical distribution of most interest is the cluster mass function 


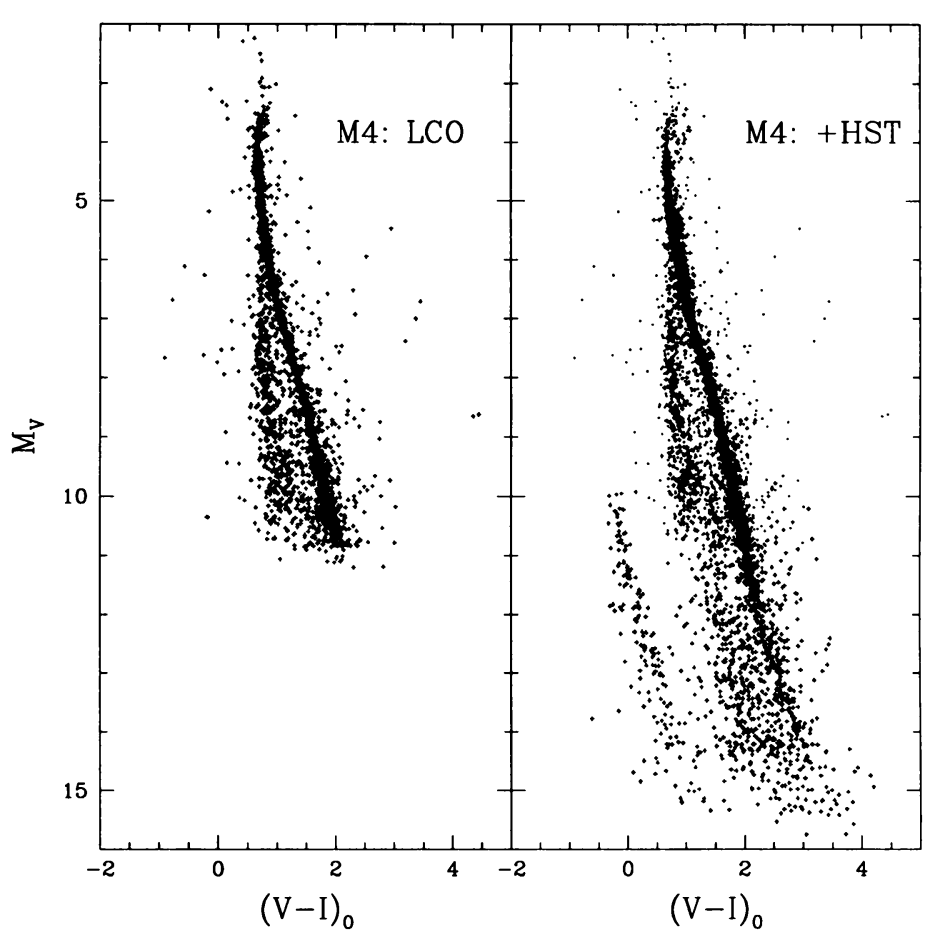

Figure 4. On the left is a ground based CMD obtained at Las Campanas Observatory (LCO) in 1990. On the right, we have added to the LCO data, our calibrated HST data from the 1 core and 4 core fields. See figure 5 for the HST data by itself on an apparent magnitude scale. The LCO field overlaps the 4 core HST field but covers about 3 times the area of the combined HST data accounting for the marked difference in the number of stars in the common magnitude region

(MF). This is obtained by multiplying the m-s LF by the slope of the luminosity-mass relationship which, in the absence of any empirical data, depends entirely on models for the cluster stars. Since adequate models are not yet in hand, we will defer a quantitative discussion of the cluster MF at this time. If the luminosity of the models is accepted, then figure 5 shows that our HST observations fall short by only a few thousandths of a solar mass from reaching the end of the end of the hydrogen burning sequence. As this limit is approached, the slope of the luminosity-mass relationship becomes extremely steep, especially in these visible photometric bands, and the $\mathrm{m}$-s becomes very sparse at the faint end. Hence the scarcity of faint $\mathrm{m}$-s stars in our fields does not necessarily imply a turn-over in the cluster MF before the end of the hydrogen burning (luminous) sequence.

We have also drawn on figure 5 the cooling locus for $0.5 \mathrm{M}_{\odot}$ wds with an 


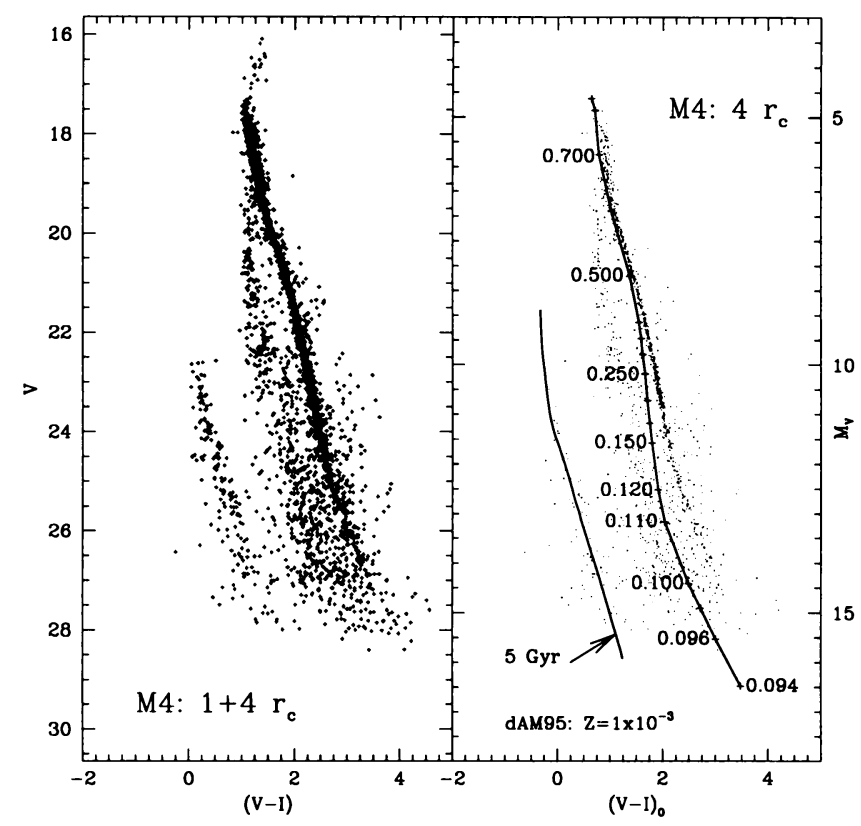

Figure 5. On the left, we show the CMD for our 1 core and 4 core fields in the apparent color-magnitude plane. The right hand panel shows the 4 core field data with a $10 \mathrm{Gyr}$. isochrone from d'Antona and Mazzitelli (1995) superimposed. We have labeled selected points along the isochrone with the mass of the model. The right hand ordinate is the absolute magnitude. Also shown is the cooling locus for a $0.5 \mathrm{M}_{\odot} \mathrm{C}-\mathrm{O}$ wd with the cooling time to reach the bottom of the observed sequence marked.

equal mix of Carbon and Oxygen in the interior. The faintest wds observed have a cooling age of about $5 \mathrm{Gyr}-\mathrm{a}$ result which is fairly insensitive to the details of the interior composition. This underscores a very important point: that the observed wds place constraints on the cluster age which are independent of the age assigned from the nuclear burning turn-off stars. With the present data, this age constraint on M4 is not particularly noteworthy. It is feasible to acquire deeper data and this could place much more interesting limits ( $\geq 10 \mathrm{Gyr}$, say) which would then bear on a number of fundamental problems in astrophysics.

\section{Discussion}

The refurbished HST has clearly lived up to its promise to reveal at last the globular cluster white dwarf cooling sequence and to extend our knowledge to encompass the entire hydrogen burning main-sequence. Our photometry 
in M4 has verified the basic theoretical tenets of white dwarf formation and evolution in the globular clusters. We have shown here that dynamical processes very likely affect the observed white dwarf cumulative luminosity function. Ultimately, this may help constrain models, like those of Drukier (1995), which incorporate stellar evolution into the Fokker-Planck framework.

It is unfortunate that the currently available model isochrones do not match the observed lower main-sequence. This problem, which may be largely due to deficiencies in the model atmospheres, will undoubtably be solved soon now that HST data is becoming available to challenge the theorists. The luminosity functions we observed in M4 still require completeness corrections but taking them as observed, and using the current, albeit imperfect, models as a guide, our data does suggest that the cluster mass function is rising to the limit of our data. There is no evidence available at this time that would suggest the cluster main-sequence is truncated before the hydrogen burning limit is reached. Hence it is very likely that the globular clusters contain a dark, brown dwarf population in addition to the cool, faint white dwarfs.

\section{References}

Allard, F. \& Hauschildt, P.M. (1995) ApJ 445, 433.

Baraffe. I., Chabrier, G., Allard, F., \& Hauschildt, P.M. (1995) ApJ 446, L35.

Bergeron, P., Wesemael, F., \& Beauchamp, A. (1996) PASP, in preparation.

Bessell, M. D., \& Brett, J. M. PASP 100, 1134.

d'Antona, F. and Mazzitelli, I (1995), preprint.

Drukier, G. A. (1995) ApJS 100, 347.

Djorgovski, S. (1993) in Structure and Dynamics of Globular Clusters, eds. S.G. Djorgovski and G. Meylan, ASP, San Francisco, p. 373.

Holtzman, J.A., Burrows, C.J., Casertano, S., Hester, J.J., Trauger, J.T., Watson, A.M., $\&$ Worthey, G. (1995) $A J$, in press.

Landolt, A.U. (1992) $A J \mathbf{1 0 4}, 340$.

Mathis, J. S. $A R A \& A$ 28, 37.

Richer, H.B., and Fahlman, G.G. (1988) ApJ 325, 218.

Richer, H.B., Fahlman, G.G., Ibata, R.I., Stetson, P.B., Bell, R.A., Bolte, M., Bond, H.E., Harris, W.E., Hesser, J.E., Mandushev, G., Pryor, C., \& VandenBerg, D.A. (1995a) ApJL 451, L17. (paper I)

Richer, H.B., Fahlman, G.G., Ibata, R.I., Ivanans, N.C., Manduschev, G., Hesser, J.E., Stetson, P.B., VandenBerg, D.A., Pryor, C., Harris, W.E., Bond, H.E., Bolte, M., \& Bell, R.A. (1995b) in The Origins, Evolution and Destinies of Binaries in Clusters, ed. E.F. Milone, in press. (paper II)

Stetson, P.B. (1994) PASP 106, 250.

Thompson, I.B., Sivaramakrishnan, A, Fahlman, G.G., \& Richer, H.B. (1990) unpublished.

Trager, S.C., King, I.R., \& Djorgovski, S. (1995) $A J$, 109, 218.

Webbink, R.F. (1985) in Dynamics of Star Clusters, IAU Symposium No. 113, eds. J. Goodman and P. Hut, Reidel, Dordrecht, p. 541.

Wood, M. A. (1992) ApJ 386, 539. 